\title{
Trinification can explain the di-photon and di-boson LHC anomalies
}

\author{
Giulio Maria Pelaggi, ${ }^{a}$ Alessandro Strumia ${ }^{a, b}$ and Elena Vigiani ${ }^{a}$ \\ ${ }^{a}$ Dipartimento di Fisica dell'Università di Pisa and INFN, \\ Largo B. Pontecorvo 3, Pisa, Italy \\ ${ }^{b}$ CERN, Theory Division, \\ 1211 Geneve 23, Switzerland \\ E-mail: g.pelaggi@for.unipi.it, Alessandro.Strumia@cern.ch, \\ elenavigiani@gmail.com
}

ABSTRACT: LHC data show a diphoton excess at $750 \mathrm{GeV}$ and a less significant diboson excess around $1.9 \mathrm{TeV}$. We propose trinification as a common source of both anomalies. The $1.9 \mathrm{TeV}$ excess can be produced by the lightest extra vector: a $W_{R}^{ \pm}$with a gauge coupling $g_{R} \approx 0.44$ that does not decay into leptons. Furthermore, trinification predicts extra scalars. One of them can reproduce the $\gamma \gamma$ excess while satisfying constraints from all other channels, given the specific set of extra fermions predicted by trinification.

KeYwords: Beyond Standard Model, Heavy Quark Physics, Higgs Physics

ARXiv EPrint: 1512.07225 


\section{Contents}

1 Introduction 1

2 Trinification 2

3 The di-boson excess at $1.9 \mathrm{TeV} \quad 4$

3.1 Trinification predictions 5

3.2 Comparison with data 7

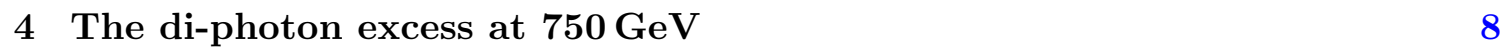

4.1 Trinification predictions 9

$\begin{array}{lll}4.2 & \text { Comparison with data } & 10\end{array}$

5 Conclusions 10

\section{Introduction}

After the discovery of the Standard Model Higgs boson [1, 2] at the Large Hadron Collider, data indicate the presence of other excesses that can be interpreted as resonant production of new bosons. The two most notable anomalies found in present LHC data are:

1. An excess of di-boson events peaked around $1.9 \mathrm{TeV}$, found in run 1 data [3-6]. This has been explained in terms of resonant production of extra vectors, such as $Z^{\prime}$ or $W_{R}$ that decay into $Z Z$ or $W Z$, respectively [7-21]. Run 2 data do not confirm this excess, but (as we will see) do not significantly contradict it.

2. An excess of di-gamma events peaked around $750 \mathrm{GeV}$, mostly seen at run 2 [4-6]. This has been explained in terms of resonant production of an extra scalar $S$ [22-42]. In order to achieve the needed $S \rightarrow \gamma \gamma$ decay width, extra vector-like fermions or scalars coupled to $S$ with a relatively large coupling must be introduced [22-42].

Assuming that these excesses are not statistical fluctuations, we try to go beyond phenomenological considerations and explore which kind of bigger picture they can suggest.

The structure needed to explain both anomalies is present in models that extend the SM gauge group around the weak scale, thereby implying extra vectors, predicting a larger set of anomaly-free chiral fermions and needing extra Higgs scalars and Yukawa couplings. Among this class of models, trinification is the $\mathrm{SU}(3)_{L} \otimes \mathrm{SU}(3)_{R} \otimes \mathrm{SU}(3)_{c}$ subgroup of $\mathrm{E}_{6}$ that explains the observed quantised hypercharges, that can be broken to the Standard Model at a scale $V$ just above the weak scale, that allows a totally asymptotically free extension of the SM [43-47], which is suggested by non conventional ideas about naturalness of the Higgs mass [48]. Trinification contains left-right models and 3-3-1 models as subgroups [49-53]. Focusing on trinification: 


\begin{tabular}{|c|c|c|c|c|c|}
\hline Field & \multicolumn{2}{|c|}{ spin generations } & $\mathrm{SU}(3)_{L}$ & $\mathrm{SU}(3)_{R}$ & $\mathrm{SU}(3)_{\mathrm{c}}$ \\
\hline $\mathcal{Q}_{R}=\left(\begin{array}{lll}U & D_{1} & D_{2}\end{array}\right)^{1}$ & $1 / 2$ & 3 & 1 & 3 & $\overline{3}$ \\
\hline $\mathcal{Q}_{L}=\left(Q \bar{D}^{\prime}\right)^{\prime}$ & $1 / 2$ & 3 & $\overline{3}$ & 1 & 3 \\
\hline $\mathcal{L}=\left(\begin{array}{ccc}\bar{L}^{\prime} & L_{2} & L_{1} \\
E & N_{2} & N_{1}\end{array}\right)$ & $1 / 2$ & 3 & 3 & $\overline{3}$ & 1 \\
\hline $\mathcal{H}=\left(\begin{array}{ccc}H_{u}^{*} & H_{d} & H_{L} \\
S^{+} & S_{1} & S_{2}\end{array}\right.$ & 0 & 3 & 3 & $\overline{3}$ & 1 \\
\hline
\end{tabular}

Table 1. Field content of minimal weak-scale trinification. $Q$ is the SM left-handed quark doublet, $U$ is the SM right-handed up quark, $E$ is the SM right-handed lepton. The $D_{1}$ and $D_{2}$ quarks mix making $D$ (right-handed down quarks) and $D^{\prime}$ (that gets a mass with $\bar{D}^{\prime}$ ) the $L_{1}$ and $L_{2}$ lepton doublets mix making $L$ (left-handed lepton doublet) and $L^{\prime}$ (that gets a mass with $\bar{L}^{\prime}$ ). $N_{1}$ and $N_{2}$ are singlets.

- It predicts extra $W_{R}^{ \pm}$vectors with mass $\sim g_{R} V$ with properties compatible with the di-boson anomaly: no decays into leptons, appropriate value of its $g_{R}$ gauge coupling.

- It predicts 27 chiral fermions per generation: the SM fermions, plus extra vector-like $D^{\prime}$ and $L^{\prime}$ fermions (and singlets), which receive masses $M^{\prime} \sim y^{\prime} V$. Large Yukawa couplings $y^{\prime}$ to fermions are needed in order to make $M^{\prime}$ above present bounds, if $V$ is not much above the weak scale.

- It predicts a larger set of Higgs scalars, which contain various singlets and doublets (with neutral components). One of them can be identified with the Higgs doublet at $125 \mathrm{GeV}$, and another with the scalar $S$ at $750 \mathrm{GeV}$.

In section 2 we briefly summarise the main ingredients of trinification models needed to interpret the LHC anomalies. In section 3 we discuss how trinification can reproduce the di-boson anomaly. In section 4 we discuss how trinification can reproduce the di-gamma anomaly. Conclusions are given in section 5 .

\section{Trinification}

The minimal model of weak-scale trinification is obtained including 3 families of chiral-free fermions under the gauge group $G_{333}=\mathrm{SU}(3)_{L} \otimes \mathrm{SU}(3)_{R} \otimes \mathrm{SU}(3)_{c}$ and 3 families of Higgses, as summarised in table 1 . The three trinification gauge coupling constants $\left(g_{2}\right.$, $\left.g_{R}, g_{3}\right)$ allow to reproduce those of the $\mathrm{SM}\left(g_{2}, g_{Y}, g_{3}\right)$ as

$$
g_{R}=\frac{2 g_{2} g_{Y}}{\sqrt{3 g_{2}^{2}-g_{Y}^{2}}} \approx 0.444 .
$$

The SM Yukawa couplings are obtained from the $G_{333}$-invariant interactions

$$
-\mathscr{L}_{Y}=y_{Q n}^{i j} \mathcal{Q}_{L i} \mathcal{Q}_{R j} \mathcal{H}_{n}+\frac{y_{L n}^{i j}}{2} \mathcal{L}_{i} \mathcal{L}_{j} \mathcal{H}_{n}+\text { h.c. }
$$



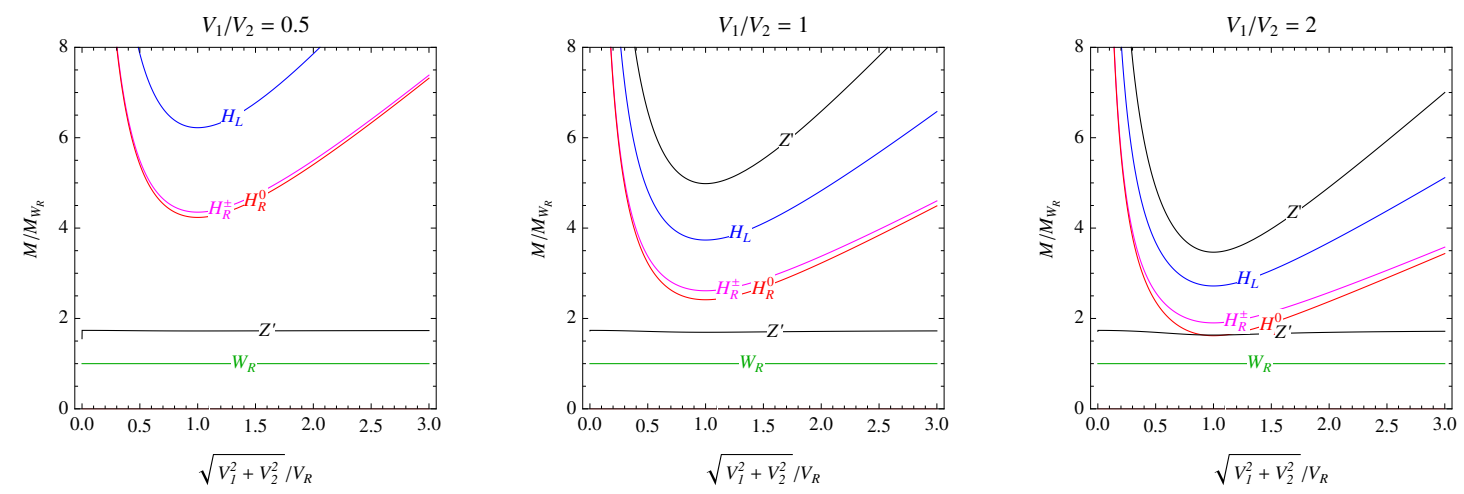

Figure 1. Trinification heavy vector masses normalised to the $W_{R}$ mass. The next-to-lightest vector is a $Z^{\prime}$ with mass $\approx 1.7 M_{W_{R}}$. For concreteness, we used a basis where $V_{R 1}=0$.

The most generic vacuum expectation values that give the desired pattern of symmetry breaking are

$$
\left\langle\mathcal{H}_{n}\right\rangle=\left(\begin{array}{ccc}
v_{u n} & 0 & 0 \\
0 & v_{d n} & v_{L n} \\
0 & V_{R n} & V_{n}
\end{array}\right) .
$$

Defining $V^{2} \equiv \sum_{n}\left(V_{n}^{2}+V_{R n}^{2}\right)$ and the dimension-less ratios

$$
\alpha \equiv \sum_{n} V_{R n}^{2} / V^{2}, \quad \beta \equiv \sum_{n} V_{n} V_{R n} / V^{2},
$$

in the relevant limit $v \ll V$ one obtains the following extra massive vectors: ${ }^{1}$

- A left-handed weak doublet with mass $M_{H_{L}}=g_{2} V / \sqrt{2}$, that contains two neutral components and two charged components.

- Two $Z^{\prime}, Z^{\prime \prime}$ vectors with masses:

$$
M_{Z^{\prime}, Z^{\prime \prime}}^{2}=\frac{V^{2}}{3}\left[\left(g_{2}^{2}+g_{R}^{2}\right) \pm \sqrt{\left(g_{2}^{2}+g_{R}^{2}\right)^{2}+3 g_{R}^{2}\left(4 g_{2}^{2}+g_{R}^{2}\right)\left(\alpha^{2}-\alpha+\beta^{2}\right)}\right] .
$$

- A SU $(2)_{R}$ vector doublet $A_{2 R}$ splitted into two neutral components with mass $M_{H_{R}^{0}}=$ $g_{R} V / \sqrt{2}$ and into 2 charged components with mass

$$
M_{H_{R}^{ \pm}}^{2}=\frac{g_{R}^{2} V^{2}}{4}\left[1+\sqrt{(1-2 \alpha)^{2}+4 \beta^{2}}\right] .
$$

${ }^{1}$ Notice that trinification includes both left-right models (the group $\mathrm{SU}(2)_{L} \otimes \mathrm{SU}(2)_{R} \otimes \mathrm{U}(1)_{B-L} \otimes \mathrm{SU}(3)_{c}$ is obtained in the limit $\alpha, \beta \ll 1[54]$ ) and 3-3-1 models [49-53] (the group $\mathrm{SU}(3)_{L} \otimes \mathrm{SU}(3)_{c} \otimes \mathrm{U}(1)_{X}$ is obtained from trinification if $\mathrm{SU}(3)_{R}$ is broken to its $\mathrm{U}(1)$ component that commutes with $\mathrm{SU}(2)_{R}$. This can be achieved adding an extra Higgs scalar in the adjoint). 3-3-1 models have the same minimal set of chiral fermions as trinification, but do not have the $W_{R}$ vector that can fit the di-boson anomaly. Minimal versions of left-right models have extra uncolored scalars but do not have extra fermions. In order to reproduce the di-gamma anomaly via gluon fusion, one can add extra quarks [55] and leptons [56], or rely on supersymmetric partners [54], or on extra components of unified multiplets which might remain light as in Alternative Left-Right Models [57]. 
- A SU $(2)_{R}$ triplet $A_{3 R}$ that splits into a neutral $Z^{\prime}$ and into 2 charged right-handed $W_{R}^{ \pm}$vectors with mass

$$
M_{W_{R}^{ \pm}}^{2}=\frac{g_{R}^{2} V^{2}}{4}\left[1-\sqrt{(1-2 \alpha)^{2}+4 \beta^{2}}\right] .
$$

We see that $W_{R}$ is the lightest extra vector. The $W_{R}^{ \pm}$and $H_{R}^{ \pm}$mass eigenstates arise as

$$
\left(\begin{array}{c}
W_{R}^{ \pm} \\
H_{R}^{ \pm}
\end{array}\right)=\left(\begin{array}{cc}
\cos \theta_{R} & -\sin \theta_{R} \\
\sin \theta_{R} & \cos \theta_{R}
\end{array}\right)\left(\begin{array}{c}
A_{R 3}^{ \pm} \\
A_{R 2}^{ \pm}
\end{array}\right)
$$

in terms of the $G_{333}$ interaction eigenstates $A_{R 3}^{ \pm}$and $A_{R 2}^{ \pm}$. The mixing angle vanishes if $\beta \ll 1$ :

$$
\tan 2 \theta_{R}=\frac{2 \beta}{1-2 \alpha} .
$$

Concerning fermions, trinification adds to the chiral fermions under the SM group $(Q, D$, $U, L$ and $E$ in the usual notation), an extra vector-like $D^{\prime} \oplus \bar{D}^{\prime}$ and $L^{\prime} \oplus \bar{L}^{\prime}$ as well as some singlets $N, N^{\prime}$. In presence of three Higgs multiplets $\mathcal{H}$, the theory has enough Yukawa couplings that the extra states can be naturally heavy, $M_{D^{\prime}, L^{\prime}} \sim y_{Q, L} V$ thanks to large enough Yukawa couplings $y_{Q, L}[43-46]$. The light and heavy fermion mass eigenstates can be parametrised as

$$
\left(\begin{array}{c}
D \\
D^{\prime}
\end{array}\right)=\left(\begin{array}{cc}
\cos \theta_{D} & \sin \theta_{D} \\
-\sin \theta_{D} & \cos \theta_{D}
\end{array}\right)\left(\begin{array}{c}
D_{1} \\
D_{2}
\end{array}\right), \quad\left(\begin{array}{c}
L \\
L^{\prime}
\end{array}\right)=\left(\begin{array}{cc}
\cos \theta_{L} & \sin \theta_{L} \\
-\sin \theta_{L} & \cos \theta_{L}
\end{array}\right)\left(\begin{array}{l}
L_{1} \\
L_{2}
\end{array}\right)
$$

where $\theta_{D}$ and $\theta_{L}$ are (for each generation) mixing angles, that can be of order one, computable in terms of Yukawa couplings and vevs. Then, the $W_{R}$ coupling to quark mass eigenstates is

$$
\frac{1}{\sqrt{2}}\left(\tilde{g}_{R} \bar{d}_{R}+\tilde{g}_{R}^{\prime} \bar{d}_{R}^{\prime}\right) W_{R}^{+} u_{R}+\text { h.c. } \quad \tilde{g}_{R}=g_{R} \cos \left(\theta_{D}+\theta_{R}\right), \quad \tilde{g}_{R}^{\prime}=g_{R} \sin \left(\theta_{D}+\theta_{R}\right),
$$

where the lightest mass eigenstate has been renamed $d_{R}$ as usual in the SM and $d_{R}^{\prime}$ represents the heavy mass eigenstate. An important feature predicted by trinification is that the $W_{R}$ does not couple to two light leptons. Rather, $W_{R}$ couples the SM doublet of left-handed leptons $L$ to the heavy doublet of anti-leptons $\bar{L}^{\prime}$ with coupling $g_{R} \sin \left(\theta_{R}-\theta_{L}\right)$, and righthanded leptons $E$ to heavy neutrinos $N_{1}, N_{2}$ with coupling $g_{R} \sin \theta_{R}, g_{R} \cos \theta_{R}$ respectively, in the limit of negligible mixing angle between neutral fermions $N_{1} / N_{2}$. Furthermore, $W_{R}$ couples to pairs of heavy leptons.

\section{The di-boson excess at $1.9 \mathrm{TeV}$}

LHC data show an excess of di-boson events around $1.9 \mathrm{TeV}$ [3]. Trinification can explain this excess as $p p \rightarrow W_{R}^{ \pm} \rightarrow W^{ \pm} Z$, and predicts an equal rate for $p p \rightarrow W_{R}^{ \pm} \rightarrow W^{ \pm} h$, some amount of $p p \rightarrow W_{R}^{ \pm} \rightarrow j j$ but no $p p \rightarrow W_{R}^{ \pm} \rightarrow \ell^{ \pm} \nu$, as discussed below. No excess is observed in di-leptons, while $j j$ data show some excess. The measured rates are summarised in table 2. Figure 2 shows the Feynman diagrams that describe such processes. 

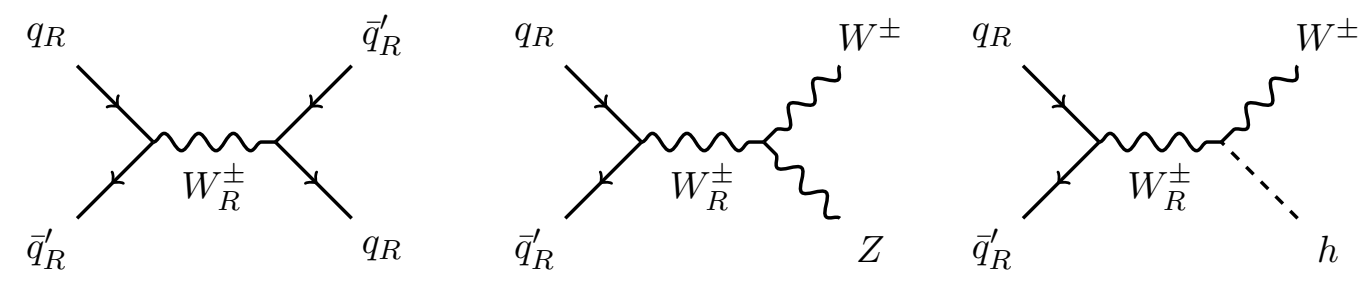

Figure 2. Resonant production of a $W_{R}^{ \pm}$that decays into $j j, W Z$ and $W h$.

\begin{tabular}{|cccccc|}
\hline process & mass & \multicolumn{2}{c}{$\sigma$ at $\sqrt{s}=8 \mathrm{TeV}$} & \multicolumn{2}{c|}{$\sigma$ at $\sqrt{s}=13 \mathrm{TeV}$} \\
\hline$p p \rightarrow S \rightarrow \gamma \gamma$ & $750 \mathrm{GeV}$ & $0.5 \pm 0.5 \mathrm{fb}$ & {$[4-6,58,59]$} & $8 \pm 2 \mathrm{fb}$ & {$[4-6]$} \\
$p p \rightarrow W_{R}^{ \pm} \rightarrow W^{ \pm} Z$ & $1.9 \mathrm{TeV}$ & $5.3 \pm 2.0 \mathrm{fb}$ & {$[3]$} & $1 \pm 13 \mathrm{fb}$ & {$[4-6,60,61]$} \\
$p p \rightarrow W_{R}^{ \pm} \rightarrow W^{ \pm} h$ & $1.9 \mathrm{TeV}$ & $3.2 \pm 3.7 \mathrm{fb}$ & {$[62-64]$} & $37 \pm 32 \mathrm{fb}$ & {$[65]$} \\
$p p \rightarrow W_{R}^{ \pm} \rightarrow j j$ & $1.9 \mathrm{TeV}$ & $73 \pm 39 \mathrm{fb}$ & {$[66-68]$} & $53 \pm 230 \mathrm{fb}$ & {$[69,70]$} \\
\hline
\end{tabular}

Table 2. Observed cross sections for the anomalous digamma and diboson excesses at LHC that we try to interpret in terms of trinification.

\subsection{Trinification predictions}

The cross section for producing a on-shell $W_{R}^{ \pm}$in narrow-width approximation is

$$
\sigma\left(p p \rightarrow W_{R}^{ \pm}\right)=K \frac{\pi \tilde{g}_{R}^{2}}{6 s} C_{ \pm}(s)
$$

where the dimensionless partonic coefficients are

$$
\begin{aligned}
& C_{+}(s)=\sum_{u, d}\left|V_{u d}\right|^{2} \int d y f_{u}\left(\frac{M_{W_{R}}}{\sqrt{s}} e^{y}\right) f_{\bar{d}}\left(\frac{M_{W_{R}}}{\sqrt{s}} e^{-y}\right), \\
& C_{-}(s)=\sum_{u, d}\left|V_{u d}\right|^{2} \int d y f_{\bar{u}}\left(\frac{M_{W_{R}}}{\sqrt{s}} e^{y}\right) f_{d}\left(\frac{M_{W_{R}}}{\sqrt{s}} e^{-y}\right),
\end{aligned}
$$

to be integrated in the rapidity range $|y|<\ln \sqrt{s} / M_{W_{R}}$, summing over all up-type and down-type quarks. The factor $K=1+8 \pi \alpha_{3}\left(M_{W_{R}}^{2}\right) / 9 \approx 1.23$ accounts for leading-order QCD corrections, with the partonic function distributions $f_{q}$ renormalised at $Q=M_{W_{R}}$. The numerical values of the $C_{ \pm}$coefficients are given in table 3 using the MSTW2008NLO partonic distribution functions [71].

The cross sections into given final states $X$ are

$$
\sigma\left(p p \rightarrow W_{R}^{ \pm} \rightarrow X\right)=\sigma\left(p p \rightarrow W_{R}^{ \pm}\right) \times \mathrm{BR}\left(W_{R}^{ \pm} \rightarrow X\right) .
$$

We then need the decay widths and the branching ratios. Simple results hold into two extreme limits, described below. 


\begin{tabular}{|c|cc|}
\hline & $\sqrt{s}=8 \mathrm{TeV}$ & $\sqrt{s}=13 \mathrm{TeV}$ \\
\hline$\sigma\left(p p \rightarrow W_{R}^{ \pm}\right)$ & $0.75 \mathrm{pb} \times \tilde{g}_{R}^{2} / \rho^{6.4}$ & $5.3 \mathrm{pb} \times \tilde{g}_{R}^{2} / \rho^{4.8}$ \\
$C_{+}(s)$ & $0.14 / \rho^{6.4}$ & $2.6 / \rho^{4.7}$ \\
$C_{-}(s)$ & $0.047 / \rho^{6.5}$ & $0.92 / \rho^{5.1}$ \\
\hline
\end{tabular}

Table 3. Cross section $\sigma\left(p p \rightarrow W_{R}^{ \pm}\right)$and partonic factors $C_{ \pm}$, as function of the $W_{R}$ mass, assumed to be close to $1.9 \mathrm{TeV}$. We defined $\rho=M_{W_{R}} / 1.9 \mathrm{TeV}$.

1. All fermions and scalars are much lighter than $M_{W_{R}}$, so that the $W_{R}$ decay width into full $G_{333}$ multiplets is simply given by

$$
\Gamma\left(W_{R}^{ \pm} \rightarrow Q_{R} \bar{Q}_{R}\right)=\Gamma\left(W_{R}^{ \pm} \rightarrow L \bar{L}\right)=2 \Gamma\left(W_{R}^{ \pm} \rightarrow \mathcal{H}^{*} \mathcal{H}\right)=\frac{g_{R}^{2}}{16 \pi} M_{W_{R}}
$$

independently of the mixing angle $\theta_{R}$ that defines $W_{R}$. Taking into account that there are 3 generations of fermions and scalars, the total width is $\Gamma_{W_{R}} / M_{W_{R}}=15 g_{R}^{2} / 32 \pi \approx$ 0.029 .

2. $W_{R}$ can only decay into the SM fermions and into the Higgs doublet, while all other states are so heavy that decays into them are kinematically forbidden. Interestingly, $W_{R}$ cannot decay into leptons. The decay width into a light quark generation is

$$
\Gamma\left(W_{R}^{+} \rightarrow u \bar{d}\right)=\frac{\tilde{g}_{R}^{2}\left|V_{u d}\right|^{2}}{16 \pi} M_{W_{R}} .
$$

The $W_{R}$ decay width into the components of the SM Higgs doublet $H=\left(\chi^{ \pm},(h+\right.$ $i \eta) / \sqrt{2}$ ) (where, after $\mathrm{SU}(2)_{L}$ breaking, $\chi^{ \pm}$and $\eta$ become the longitudinal components of $W^{ \pm}$and $Z$, respectively) is

$$
\Gamma\left(W_{R}^{ \pm} \rightarrow W^{ \pm} Z\right)=\Gamma\left(W_{R}^{ \pm} \rightarrow W^{ \pm} h\right)=\frac{g_{R}^{2}}{192 \pi} M_{W_{R}} \cos ^{2} \theta_{H}
$$

where $\theta_{H}$ is a mixing angle that defines which components of $\mathcal{H}$ contain the light SM Higgs doublet $H$, given by $\cos \theta_{H} \equiv r_{u d} \cos \theta_{R}-r_{u L} \sin \theta_{R}$ with $^{2}$

$$
r_{u d}=\frac{2 \sum_{n} v_{u n} v_{d n}}{\sum_{n}\left(v_{u n}^{2}+v_{d n}^{2}+v_{L n}^{2}\right)}, \quad r_{u L}=\frac{2 \sum_{n} v_{u n} v_{L n}}{\sum_{n}\left(v_{u n}^{2}+v_{d n}^{2}+v_{L n}^{2}\right)} .
$$

In this limit, the total decay width is $\Gamma_{W_{R}} / M_{W_{R}}=g_{R}^{2}\left(18 \cos ^{2}\left(\theta_{D}+\theta_{R}\right)+\cos ^{2} \theta_{H}\right) / 96 \pi$.

\footnotetext{
${ }^{2}$ The decay width into SM vector bosons can also be computed taking into account that the small SM vacuum expectation values $v$ mixes $W_{R}$ with the SM $W$ by a small angle

$$
\theta_{L R} \simeq \frac{g_{R}}{g_{2}} \frac{M_{W}^{2}}{M_{W_{R}}^{2}} \cos \theta_{H}
$$

and that

$$
\Gamma\left(W_{R}^{ \pm} \rightarrow W^{ \pm} Z\right)=\Gamma\left(W_{R}^{ \pm} \rightarrow W^{ \pm} h\right)=\frac{g_{2}^{2} \theta_{L R}^{2}}{192 \pi} \frac{M_{W_{R}}^{5}}{M_{W}^{4}}=\frac{g_{R}^{2}}{192 \pi} M_{W_{R}} \cos ^{2} \theta_{H}
$$



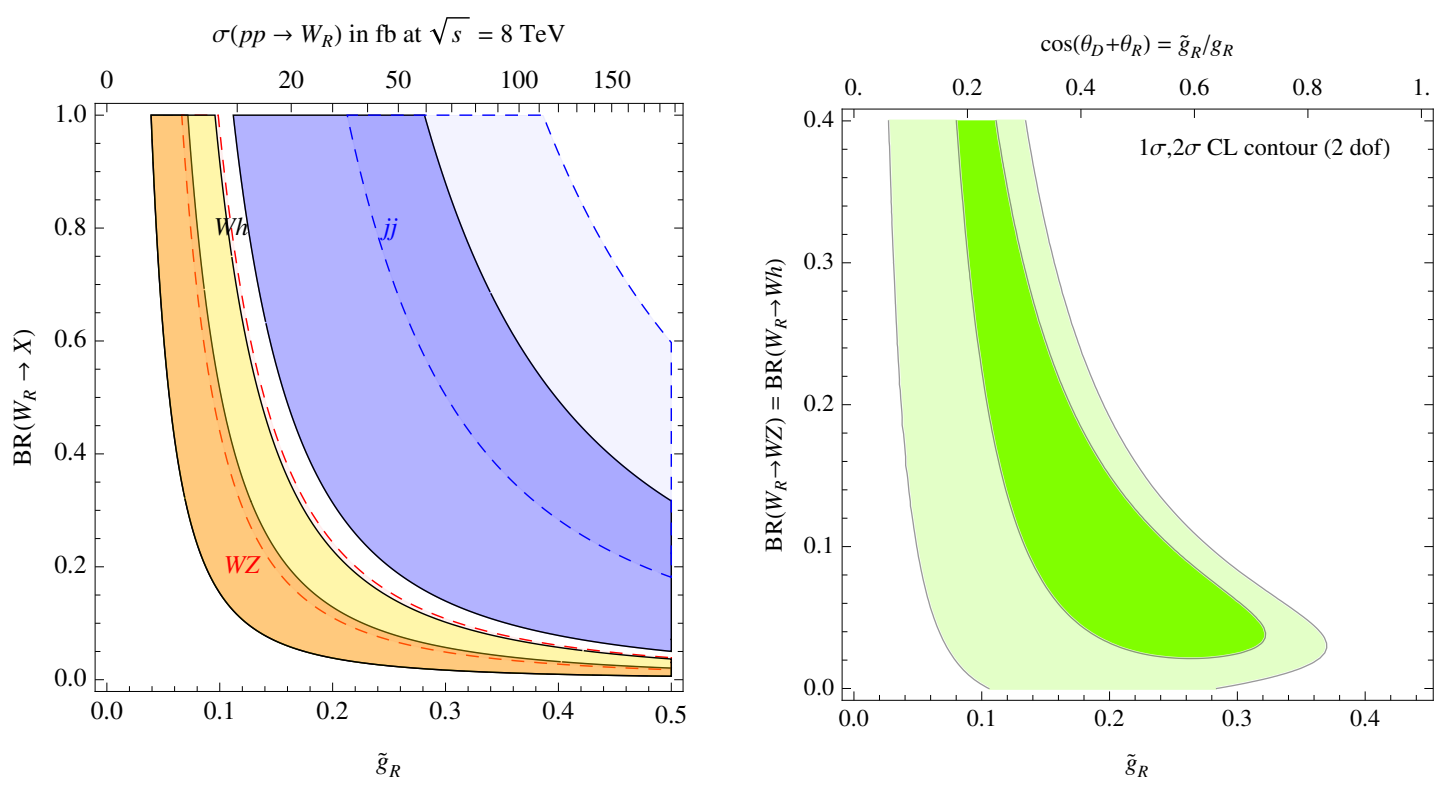

Figure 3. Global fit of run 1 and run 2 data. Left: $\pm 1 \sigma$ experimental bands for the $W_{R}$ Branching Ratios into $j j$ (blue), $W Z$ (red), $W h$ (yellow) as function of the coupling $\tilde{g}_{R}$ that controls the predicted $W_{R}$ production cross section. We also show the bands obtained using $8 \mathrm{TeV}$ data (dashed contours with lighter colors), and the cross section at $8 \mathrm{TeV}$ (upper axis). Right: global fit (green region) assuming only $W_{R}$ decays into $j j, W Z, W h$.

One loop QCD corrections enhance the decay widths into quarks by $K_{D}=1+$ $\alpha_{3}\left(M_{W_{R}}\right) / \pi \approx 1.03$.

If all the mixing factors are of order unity, in case 2 one expects $\operatorname{BR}\left(W_{R}^{ \pm} \rightarrow W^{ \pm} Z\right)=$ $\mathrm{BR}\left(W_{R}^{ \pm} \rightarrow W^{ \pm} h\right) \sim 1 / 38$. However this branching ratio can be much larger if $\cos ^{2} \theta_{H} \gg$ $\cos ^{2}\left(\theta_{D}+\theta_{R}\right)$. In case 1 one has $\operatorname{BR}\left(W_{R}^{ \pm} \rightarrow W^{ \pm} Z\right)=\mathrm{BR}\left(W_{R}^{ \pm} \rightarrow W^{ \pm} h\right) \leq 1 / 90$.

\subsection{Comparison with data}

We are now ready to compare the trinification predictions with di-boson data.

The first issue that we address is the compatibility of run 1 data at $\sqrt{s}=8 \mathrm{TeV}$ (that showed an excess around $1.9 \mathrm{TeV}$ ) with run 2 data at $\sqrt{s}=13 \mathrm{TeV}$, that show no excess so far. In view of the reduced luminosity so far accumulated at run 2, and of the larger backgrounds, run 2 data, as summarised in table 2 , have much larger uncertainties. In our model the $W_{R}$ production cross section grows by a factor $\approx 7$ when going from $\sqrt{s}=8$ to $13 \mathrm{TeV}$.

Taking into account all these factors, the incompatibility between the two data-sets is only mild, at the $1 \sigma$ level, and the run 1 data still play the main role. This is shown in the left panel of figure 3 , where we extract the $W_{R}$ branching ratios into the various channels as function of the $W_{R}$ production cross section, plotted at $8 \mathrm{TeV}$ on the upper horizontal axis, and parametrised in terms of the coupling $\tilde{g}_{R}$ on the lower axis.

We next proceed in performing a global fit, assuming that $W_{R}$ only decays into $j j, W Z$ and $W h$ (case 2 in the discussion above) and using the theoretical prediction $\operatorname{BR}\left(W_{R} \rightarrow\right.$ $W Z)=\mathrm{BR}\left(W_{R} \rightarrow W h\right)$. The result is shown in the right panel of figure 3 . We see that data 
prefer $\tilde{g}_{R} \sim 0.25$. While in a generic context this is a free parameter, trinification predicts $\tilde{g}_{R}=g_{R} \cos \left(\theta_{D}+\theta_{R}\right)$, where $g_{R}=0.444$ and the mixing factor depends on the details of the model. This means that trinification is non-trivially compatible with data, that favour a substantial amount of mixing among right-handed down quarks, $\cos \left(\theta_{D}+\theta_{R}\right) \sim 0.5$. The best fit has $\chi_{\text {best }}^{2}=\chi_{\mathrm{SM}}^{2}-2.7^{2}$, which can be interpreted as a $2.7 \sigma$ hint, up to look-elsewhere reductions. Similar results are obtained for a slightly lighter or heavier $W_{R}$.

Allowing $W_{R}$ to decay into extra light states (up to the opposite extremum of case 1 in the discussion above) reduces the $W_{R}$ branching ratios into $j j, W Z, W h$, such that a higher production cross section becomes needed to fit the data. Trinification allows to raise the production cross section up to $\tilde{g}_{R}=g_{R}$. At the same time, the extra decay channels give rise to extra more complicated signals.

In order to keep the discussion simple, let us divide particles into three main categories: $q$ (generic quarks), $\ell$ (generic leptons), $H$ (Higgses: $h, W, Z$ ), all approximatively massless. Then $q^{\prime}$ denotes the extra heavy quarks, $\ell^{\prime}$ the extra heavy leptons, and $H^{\prime}$ denotes the extra heavy scalars (not eaten by the $W, Z$ ), with generic masses $M^{\prime}$. The possible $W_{R}$ decay modes are

$$
W_{R} \rightarrow \begin{cases}q q, H H & \text { always } \\ q q^{\prime}, \ell \ell^{\prime}, H H^{\prime} & \text { if } M^{\prime} \lesssim M_{W_{R}} \\ q^{\prime} q^{\prime}, \ell^{\prime} \ell^{\prime}, H^{\prime} H^{\prime} & \text { if } M^{\prime} \lesssim M_{W_{R}} / 2\end{cases}
$$

The extra primed particles, if produced, can decay through gauge interactions as

$$
q^{\prime} \rightarrow q W_{R}^{*}, \quad \ell^{\prime} \rightarrow \ell W_{R}^{*}, \quad H^{\prime} \rightarrow H W_{R}^{*}
$$

(where the virtual $W_{R}^{*}$ can, in many cases, only materialise into $q q$ or $H H$ light particles) and through Yukawa interactions as

$$
q^{\prime} \rightarrow q H, \quad \ell^{\prime} \rightarrow \ell H, \quad H^{\prime} \rightarrow q q, \ell \ell .
$$

If $W_{R} \rightarrow \ell \ell^{\prime}$ is kinematically allowed, one obtains final states with two leptons accompanied by a $j j$ or $W Z$ or $W h$ pair or by a single $W, Z, h$. If $\ell^{\prime}$ is a heavy neutrino, Majorana masses can give rise to same-sign di-leptons, accompanied by the same extra particles listed above. In particular, for appropriate values of its parameters, trinification can also reproduce the eejj excess [72], as already discussed in [7-21] in the context of dedicated models. Needless to say, all decay widths can be computed in terms of unknown masses and of the mixing angles introduced above.

\section{The di-photon excess at $750 \mathrm{GeV}$}

The di-photon excess at $750 \mathrm{GeV}$ [4-6] has been tentatively interpreted as a new scalar $S$ with mass $M \approx 750 \mathrm{GeV}$ that is produced as $g g \rightarrow S$ and decays as $S \rightarrow \gamma \gamma$ through loops of extra fermions and/or scalars coupled to $S$ by sizeable couplings [22-42]. ATLAS data [4$6]$ possibly indicate a large width $\Gamma_{S} / M \approx 0.06$, which would make this interpretation more difficult. In any case, the apparently large width can be faked by a multiplet of quasi-degenerate narrow resonances [22]. 
Trinification has built-in all the ingredients needed to provide such interpretation: extra scalars $S$ as singlets and/or doublets in the Higgs $\mathcal{H}$ multiplets; extra fermions $D^{\prime}$ and $L^{\prime}$ that extend the SM chiral fermion content and receive masses only as $y V$; Yukawa couplings $y$ that must be sizeable enough in order to push the extra fermions above the experimental bounds.

\subsection{Trinification predictions}

In order to compute the relevant decay widths of $S$ into $\gamma \gamma$ and $g g$, we parameterise its linear Lagrangian couplings to generic fermions $\mathcal{Q}_{f}$ with Dirac mass $M_{f}$, to generic scalars $\tilde{\mathcal{Q}}_{s}$ with mass $M_{s}$ and to generic vectors $V$ with mass $M_{V}$ as

$$
S \overline{\mathcal{Q}}\left(y_{f}+y_{5 f} \gamma_{5}\right) \mathcal{Q}-S A_{s}\left|\tilde{\mathcal{Q}}_{s}\right|^{2}+g_{V} M_{V} S\left|V_{\mu}\right|^{2} .
$$

The coupling $g_{V}$ is normalised such that it coincides with the SM coupling $g_{2}$, if $S$ is replaced by the SM Higgs $h$ and $V_{\mu}$ by the SM $W_{\mu}^{ \pm}$. Then $S$ acquires the following decay widths at one-loop level [22]:

$$
\begin{aligned}
\frac{\Gamma(S \rightarrow \gamma \gamma)}{M}= & \frac{\alpha^{2}}{256 \pi^{3}}\left(\left|\sum_{f} \frac{y_{5 f} M}{M_{f}} Q_{f}^{2} F_{5 f}\right|^{2}+\right. \\
& \left.+\left|\sum_{f} \frac{y_{f} M}{M_{f}} Q_{f}^{2} F_{f}+\sum_{s} \frac{A_{s} M}{2 M_{s}^{2}} Q_{s}^{2} F_{s}-\sum_{V} \frac{g_{V} M}{2 M_{V}} Q_{V}^{2} F_{V}\right|^{2}\right), \\
\frac{\Gamma(S \rightarrow g g)}{M}= & \frac{\alpha_{3}^{2}}{32 \pi^{3}}\left(\left|\sum_{f} C_{f} \frac{y_{5 f} M}{M_{f}} F_{5 f}\right|^{2}+\left|\sum_{f} C_{f} \frac{y_{f} M}{M_{f}} F_{f}\right|^{2}\right),
\end{aligned}
$$

where multiplicities are implicitly included in sums. The color factors are $C_{1}=0$ for color singlets and $C_{3}=1 / 2$ for color triplets, and we have taken into account that trinification predicts no other coloured particles. The loop functions are [22]

$$
\begin{aligned}
& F_{s}=x[-1+x f(x)] \quad \stackrel{x \rightarrow \infty}{=} 1 / 3 \text { with } x=4 M_{s}^{2} / M^{2}, \\
& F_{f}=2 x[1+(1-x) f(x)] \quad \stackrel{x \rightarrow \infty}{=} 4 / 3 \text { with } x=4 M_{f}^{2} / M^{2}, \\
& F_{5 f}=2 x f(x) \quad \stackrel{x \rightarrow \infty}{=} 2 \quad \text { with } x=4 M_{f}^{2} / M^{2}, \\
& F_{V}=2+3 x+3 x(2-x) f(x) \stackrel{x \rightarrow \infty}{=} 7 \quad \text { with } x=4 M_{V}^{2} / M^{2} \text {, }
\end{aligned}
$$

where $f(x)=\arctan ^{2}(1 / \sqrt{x-1})$. The $S$ decay width into gluons can be estimated as

$$
\frac{\Gamma(S \rightarrow g g)}{M} \approx 0.610^{-4}\left(y_{5 D}^{2}+\frac{4}{9} y_{D}^{2}\right)\left(\frac{1 \mathrm{TeV}}{M_{D^{\prime}}} \frac{N_{D^{\prime}}}{3}\right)^{2}
$$

having assumed, for simplicity, $N_{D^{\prime}} \leq 3$ degenerate copies of $D^{\prime}$ with mass $M_{D^{\prime}} \gg M / 2$. This shows that the $\Gamma(S \rightarrow g g) / M \gtrsim 10^{-5}$ needed to fit the di-gamma anomaly [22] is easily achieved; furthermore trinification implies that some components of the $\mathcal{H}$ scalars have sizeable couplings to SM quarks, allowing for extra production channels of $S$. The $S$ decay width into photons can be estimated as

$$
\frac{\Gamma(S \rightarrow \gamma \gamma)}{M} \approx 10^{-6}\left[6.7\left(y_{5 E} \frac{N_{L^{\prime}}}{3}\right)^{2}+\left(1.05 y_{E} \frac{N_{L^{\prime}}}{3}+1.15 \frac{A_{s}}{M_{s}} \frac{N_{s}}{9}\right)^{2}\right]
$$


having assumed, for simplicity, $N_{L^{\prime}} \leq 3$ copies of leptons degenerate at $M_{L^{\prime}} \gtrsim M / 2$, plus $N_{s} \leq 9$ copies of scalars with charge $Q_{s}= \pm 1$ degenerate at the same mass and with the same cubic $A_{s}$. We neglected the small contribution from heavy down-quarks and from vectors.

\subsection{Comparison with data}

In order to achieve the $\Gamma(S \rightarrow \gamma \gamma) / M \gtrsim 10^{-6}$ needed to reproduce the $\gamma \gamma$ anomaly [22] one either needs Yukawa couplings of order unity or larger, or the presence of multiple charged states, or a large $A_{s} / M$.

In a context where the theory can be extrapolated up to infinite energy [47, 48], some scalar quartic couplings can be predicted in terms of squared gauge couplings, times modeldependent order one factors. This implies that scalars typically have masses comparable to vectors, which must be heavier than about $2 \mathrm{TeV} .{ }^{3}$ One can assume that one of the various scalar doublets contained in $\mathcal{H}_{1,2,3}$ is accidentally lighter and can be identified with the $125 \mathrm{GeV}$ Higgs boson, and that one singlet (or doublet) is accidentally light and can be identified with the $750 \mathrm{GeV}$ resonance. In such a case, the accidental cancellation that makes its mass $M$ smaller than $V$ does not generically suppress, at the same time, its cubic couplings $A_{s}$, such that $A_{s} / M$ can be large (phenomenologically limited by vacuum decay constraints) enhancing $S \rightarrow \gamma \gamma$. The mixing of $S$ with the physical Higgs $h$ must be small enough. The two neutral components of a doublet generically receive a small mass splitting $\sim v^{2} / M \approx 10 \mathrm{GeV}$ and can mimic the large width favoured by ATLAS data [22]. The two components of all electroweak singlets in $\mathcal{H}$ generically receive mass splittings of order $V$.

If the $S \rightarrow \gamma \gamma$ process is dominated by a loop of either lepton doublets or scalar doublets (which have the same quantum numbers) one predicts the following extra electroweak decays:

$$
\frac{\Gamma(S \rightarrow Z \gamma)}{\Gamma(S \rightarrow \gamma \gamma)} \approx 0.9<2, \quad \frac{\Gamma(S \rightarrow Z Z)}{\Gamma(S \rightarrow \gamma \gamma)} \approx 3.5<6, \quad \frac{\Gamma(S \rightarrow W W)}{\Gamma(S \rightarrow \gamma \gamma)} \approx 10<20 .
$$

These predictions satisfy the experimental bounds collected in [22] and quoted after the < symbols and correspond, in the language of effective operators of [22], to equal coefficients $\Lambda_{B}=\Lambda_{W}$, or $\tilde{\Lambda}_{B}=\tilde{\Lambda}_{W}$. The extra loop contribution from charged scalar singlets or from right-handed down quarks can reduce the ratios in eq.(4.5).

\section{Conclusions}

Models based on the trinification gauge group $G_{333}=\mathrm{SU}(3)_{L} \otimes \mathrm{SU}(3)_{R} \otimes \mathrm{SU}(3)_{c}$ explain the quantisation of electric charge [43-46]. Weak scale trinification models provide natural

\footnotetext{
${ }^{3}$ An exception arises if the breaking of $G_{333}$ proceeds through the Coleman-Weinberg mechanism. Then the scalar singlet that corresponds to a dilatation of all vacuum expectation values $V$ remains lighter than the other ones by a loop factor. If this dilaton is the $S$ state, one obtains more neat predictions, given that vectors and fermions acquire mass only from its vev $\langle S\rangle=V$, such that $M_{V}=g_{V} V / 2, M_{f}=y_{f} V, y_{5 f}=0$. However, in such a case, $\Gamma(S \rightarrow \gamma \gamma)$ is somehow too small, given that such dilaton cannot have a large cubic $A_{s}$, and that $V \gtrsim$ few $\mathrm{TeV}$.
} 
extensions of the Standard Model that can hold up to infinite energy, with all gauge and Yukawa and quartic couplings flowing to asymptotic freedom [47].

We have shown that these same models, without any extra ingredient, can simultaneously explain the $\gamma \gamma$ anomaly at $750 \mathrm{GeV}$ (section 4) and the diboson anomaly at $1.9 \mathrm{TeV}$ (section 3). Run 2 LHC data do not confirm the latter anomaly found in run 1 data, but do not significantly disfavour it (figure 3a).

The lightest extra vector of trinification is a $W_{R}^{ \pm}$with a gauge coupling $g_{R} \approx 0.444$ compatible with the cross section needed to fit the $1.9 \mathrm{TeV}$ anomaly (figure $3 \mathrm{~b}$ ). Furthermore, the group structure of trinification implies that it does not decay to SM leptons, in agreement with data.

At the same time, trinification predicts a set of extra fermions (chiral under $G_{333}$ but not under the SM) and of scalars which can be numerous and light enough to mediate the $g g \rightarrow S \rightarrow \gamma \gamma$ loops without giving, at the same time, excessive $g g \rightarrow S \rightarrow \gamma Z, Z Z, W^{+} W^{-}$. This interplay between the 2 anomalies implies extra $W_{R}$ decay channels which give rise to the extra signals discussed in section 3 .

\section{Acknowledgments}

We thank the ATLAS and CMS colleagues for their beautiful Christmas gift, hoping that with the restart of data taking on April 1 it will not become a bad joke. We thank R. Franceschini, M. Redi, J.F. Kamenik M.McCullogh for useful discussions. This work was supported by the ERC grant NEO-NAT.

Open Access. This article is distributed under the terms of the Creative Commons Attribution License (CC-BY 4.0), which permits any use, distribution and reproduction in any medium, provided the original author(s) and source are credited.

\section{References}

[1] ATLAS collaboration, Observation of a new particle in the search for the standard model Higgs boson with the ATLAS detector at the LHC, Phys. Lett. B 716 (2012) 1 [arXiv: 1207.7214] [INSPIRE].

[2] CMS collaboration, Observation of a new boson at a mass of $125 \mathrm{GeV}$ with the CMS experiment at the LHC, Phys. Lett. B 716 (2012) 30 [arXiv:1207.7235] [INSPIRE].

[3] F. Dias et al., Combination of Run-1 exotic searches in diboson final states at the LHC, arXiv: 1512.03371 [INSPIRE].

[4] J. Olsen and M. Kado, ATLAS and CMS physics results from Run 2, talk at LHC seminar, CERN, Geneva Switzerland December 152015.

[5] ATLAS collaboration, Search for resonances decaying to photon pairs in $3.2 \mathrm{fb}^{-1}$ of $p p$ collisions at $\sqrt{s}=13 \mathrm{TeV}$ with the ATLAS detector, ATLAS-CONF-2015-081, CERN, Geneva Switzerland (2015).

[6] CMS collaboration, Search for new physics in high mass diphoton events in proton-proton collisions at $\sqrt{s}=13 \mathrm{TeV}$, CMS-PAS-EXO-15-004, CERN, Geneva Switzerland (2015). 
[7] J. Hisano, N. Nagata and Y. Omura, Interpretations of the ATLAS diboson resonances, Phys. Rev. D 92 (2015) 055001 [arXiv: 1506.03931] [InSPIRE].

[8] K. Cheung, W.-Y. Keung, P.-Y. Tseng and T.-C. Yuan, Interpretations of the ATLAS diboson anomaly, Phys. Lett. B 751 (2015) 188 [arXiv:1506.06064] [INSPIRE].

[9] B.A. Dobrescu and Z. Liu, $W^{\prime}$ boson near 2 TeV: predictions for run 2 of the LHC, Phys. Rev. Lett. 115 (2015) 211802 [arXiv:1506.06736] [INSPIRE].

[10] Y. Gao, T. Ghosh, K. Sinha and J.-H. Yu, SU(2) $\times \mathrm{SU}(2) \times \mathrm{U}(1)$ interpretations of the diboson and Wh excesses, Phys. Rev. D 92 (2015) 055030 [arXiv:1506.07511] [INSPIRE].

[11] J. Brehmer, J. Hewett, J. Kopp, T. Rizzo and J. Tattersall, Symmetry restored in dibosons at the LHC?, JHEP 10 (2015) 182 [arXiv:1507.00013] [INSPIRE].

[12] Q.-H. Cao, B. Yan and D.-M. Zhang, Simple non-Abelian extensions of the standard model gauge group and the diboson excesses at the LHC, Phys. Rev. D 92 (2015) 095025 [arXiv: 1507.00268] [INSPIRE].

[13] T. Abe, R. Nagai, S. Okawa and M. Tanabashi, Unitarity sum rules, three-site moose model and the ATLAS 2 TeV diboson anomalies, Phys. Rev. D 92 (2015) 055016 [arXiv: 1507.01185] [INSPIRE].

[14] P.S. Bhupal Dev and R.N. Mohapatra, Unified explanation of the eejj, diboson and dijet resonances at the LHC, Phys. Rev. Lett. 115 (2015) 181803 [arXiv:1508.02277] [INSPIRE].

[15] P. Coloma, B.A. Dobrescu and J. Lopez-Pavon, Right-handed neutrinos and the $2 \mathrm{TeV} \mathrm{W}^{\prime}$ boson, Phys. Rev. D 92 (2015) 115023 [arXiv: 1508.04129] [InSPIRE].

[16] F.F. Deppisch et al., Reconciling the $2 \mathrm{TeV}$ excesses at the LHC in a linear seesaw left-right model, Phys. Rev. D 93 (2016) 013011 [arXiv: 1508.05940] [INSPIRE].

[17] J.H. Collins and W.H. Ng, A $2 \mathrm{TeV} W_{R}$, supersymmetry and the Higgs mass, JHEP 01 (2016) 159 [arXiv:1510.08083] [INSPIRE].

[18] B.A. Dobrescu and P.J. Fox, Signals of a $2 \mathrm{TeV} W^{\prime}$ boson and a heavier $Z^{\prime}$ boson, arXiv: 1511.02148 [INSPIRE].

[19] B. Bhattacherjee, P. Byakti, C.K. Khosa, J. Lahiri and G. Mendiratta, Alternative search strategies for a BSM resonance fitting ATLAS diboson excess, arXiv: 1511.02797 [INSPIRE].

[20] K. Das, T. Li, S. Nandi and S.K. Rai, Diboson excesses in an anomaly free leptophobic left-right model, Phys. Rev. D 93 (2016) 016006 [arXiv:1512.00190] [INSPIRE].

[21] A. Das, N. Nagata and N. Okada, Testing the $2 \mathrm{TeV}$ resonance with trileptons, arXiv: 1601.05079 [INSPIRE].

[22] R. Franceschini et al., What is the $\gamma \gamma$ resonance at $750 \mathrm{GeV}$ ?, arXiv: 1512.04933 [INSPIRE].

[23] A. Angelescu, A. Djouadi and G. Moreau, Scenarii for interpretations of the LHC diphoton excess: two Higgs doublets and vector-like quarks and leptons, arXiv:1512.04921 [INSPIRE].

[24] S. Knapen, T. Melia, M. Papucci and K. Zurek, Rays of light from the LHC, arXiv: 1512.04928 [INSPIRE].

[25] D. Buttazzo, A. Greljo and D. Marzocca, Knocking on new physics' door with a scalar resonance, arXiv: 1512.04929 [INSPIRE].

[26] M. Low, A. Tesi and L.-T. Wang, A pseudoscalar decaying to photon pairs in the early LHC run 2 data, arXiv: 1512.05328 [INSPIRE]. 
[27] B. Dutta, Y. Gao, T. Ghosh, I. Gogoladze and T. Li, Interpretation of the diphoton excess at $C M S$ and $A T L A S$, arXiv: 1512.05439 [INSPIRE].

[28] Q.-H. Cao, Y. Liu, K.-P. Xie, B. Yan and D.-M. Zhang, A boost test of anomalous diphoton resonance at the $L H C$, arXiv: 1512.05542 [INSPIRE].

[29] D. Becirevic, E. Bertuzzo, O. Sumensari and R.Z. Funchal, Can the new resonance at LHC be a CP-odd Higgs boson?, arXiv:1512.05623 [INSPIRE].

[30] S. Fichet, G. von Gersdorff and C. Royon, Scattering light by light at $750 \mathrm{GeV}$ at the LHC, arXiv: 1512.05751 [INSPIRE].

[31] E. Gabrielli, K. Kannike, B. Mele, M. Raidal, C. Spethmann and H. Veermäe, A SUSY inspired simplified model for the $750 \mathrm{GeV}$ diphoton excess, arXiv:1512.05961 [INSPIRE].

[32] J. Chakrabortty, A. Choudhury, P. Ghosh, S. Mondal and T. Srivastava, Di-photon resonance around $750 \mathrm{GeV}$ : shedding light on the theory underneath, arXiv:1512.05767 [INSPIRE].

[33] A. Falkowski, O. Slone and T. Volansky, Phenomenology of a $750 \mathrm{GeV}$ singlet, JHEP 02 (2016) 152 [arXiv : 1512.05777] [INSPIRE].

[34] A. Alves, A.G. Dias and K. Sinha, The $750 \mathrm{GeV}$ S-cion: where else should we look for it?, arXiv: 1512.06091 [INSPIRE].

[35] E. Megias, O. Pujolàs and M. Quirós, On dilatons and the LHC diphoton excess, arXiv: 1512.06106 [INSPIRE].

[36] W. Chao, Symmetries behind the $750 \mathrm{GeV}$ diphoton excess, arXiv:1512.06297 [INSPIRE].

[37] S. Chang, A simple U(1) gauge theory explanation of the diphoton excess, arXiv:1512.06426 [INSPIRE].

[38] R. Ding, L. Huang, T. Li and B. Zhu, Interpreting 750 GeV diphoton excess with R-parity violation supersymmetry, arXiv:1512.06560 [INSPIRE].

[39] D. Bardhan, D. Bhatia, A. Chakraborty, U. Maitra, S. Raychaudhuri and T. Samui, Radion candidate for the LHC diphoton resonance, arXiv:1512.06674 [INSPIRE].

[40] M. Dhuria and G. Goswami, Perturbativity, vacuum stability and inflation in the light of $750 \mathrm{GeV}$ diphoton excess, arXiv: 1512.06782 [INSPIRE].

[41] X.-F. Han and L. Wang, Implication of the $750 \mathrm{GeV}$ diphoton resonance on two-Higgs-doublet model and its extensions with Higgs field, arXiv:1512.06587 [INSPIRE].

[42] J.S. Kim, K. Rolbiecki and R.R. de Austri, Model-independent combination of diphoton constraints at $750 \mathrm{GeV}$, arXiv: 1512.06797 [INSPIRE].

[43] Y. Achiman and B. Stech, Topless model for grand unification, in New phenomena in lepton-hadron physics, D.E.C. Fries and J. Wess eds., Plenum, New York U.S.A. (1979), pg. 303.

[44] A. de Rujula, H. Georgi and S.L. Glashow, Trinification of all elementary particle forces, in Fifth Workshop on Grand Unification, K. Kang, H. Fried and P. Frampton eds., World Scientific, Singapore (1984), pg. 88.

[45] J. Hetzel and B. Stech, Low-energy phenomenology of trinification: an effective left-right-symmetric model, Phys. Rev. D 91 (2015) 055026 [arXiv:1502.00919] [InSPIRE].

[46] J. Hetzel, Phenomenology of a left-right-symmetric model inspired by the trinification model, arXiv: 1504.06739 [INSPIRE]. 
[47] G.M. Pelaggi, A. Strumia and S. Vignali, Totally asymptotically free trinification, JHEP 08 (2015) 130 [arXiv: 1507.06848] [INSPIRE].

[48] G.F. Giudice, G. Isidori, A. Salvio and A. Strumia, Softened gravity and the extension of the standard model up to infinite energy, JHEP 02 (2015) 137 [arXiv:1412.2769] [INSPIRE].

[49] M. Singer, J.W.F. Valle and J. Schechter, Canonical neutral current predictions from the weak electromagnetic gauge group $\mathrm{SU}(3) \times \mathrm{U}(1)$, Phys. Rev. D 22 (1980) 738 [inSPIRE].

[50] F. Pisano and V. Pleitez, An SU(3) $\times$ U(1) model for electroweak interactions, Phys. Rev. D 46 (1992) 410 [hep-ph/9206242] [INSPIRE].

[51] W.A. Ponce, J.B. Florez and L.A. Sanchez, Analysis of $\mathrm{SU}(3)_{C} \times \mathrm{SU}(3)_{L} \times \mathrm{U}(1)_{X}$ local gauge theory, Int. J. Mod. Phys. A 17 (2002) 643 [hep-ph/0103100] [INSPIRE].

[52] W.A. Ponce, Y. Giraldo and L.A. Sanchez, Minimal scalar sector of 3-3-1 models without exotic electric charges, Phys. Rev. D 67 (2003) 075001 [hep-ph/0210026] [INSPIRE].

[53] G. Cacciapaglia, C. Csáki, G. Marandella and A. Strumia, The minimal set of electroweak precision parameters, Phys. Rev. D 74 (2006) 033011 [hep-ph/0604111] [INSPIRE].

[54] C. Hati, Explaining the diphoton excess in alternative left-right symmetric model, arXiv: 1601.02457 [INSPIRE].

[55] U.K. Dey, S. Mohanty and G. Tomar, 750 GeV resonance in the dark left-right model, arXiv:1512.07212 [INSPIRE].

[56] F.F. Deppisch, C. Hati, S. Patra, P. Pritimita and U. Sarkar, Implications of the diphoton excess on left-right models and gauge unification, arXiv:1601.00952 [INSPIRE].

[57] E. Ma, Particle dichotomy and left-right decomposition of $E_{6}$ superstring models, Phys. Rev. D 36 (1987) 274 [INSPIRE].

[58] CMS collaboration, Search for high-mass diphoton resonances in pp collisions at $\sqrt{s}=8 \mathrm{TeV}$ with the CMS detector, CMS-PAS-EXO-12-045, CERN, Geneva Switzerland (2012).

[59] ATLAS collaboration, Search for high-mass diphoton resonances in pp collisions at $\sqrt{s}=8 \mathrm{TeV}$ with the ATLAS detector, Phys. Rev. D 92 (2015) 032004 [arXiv:1504.05511] [INSPIRE].

[60] ATLAS collaboration, Search for $W W / W Z$ resonance production in the $\ell \nu q q$ final state at $\sqrt{s}=13 \mathrm{TeV}$ with the ATLAS detector at the LHC, ATLAS-CONF-2015-075, CERN, Geneva Switzerland (2015).

[61] CMS collaboration, Search for massive resonances decaying into pairs of boosted $W$ and $Z$ bosons at $\sqrt{s}=13 \mathrm{TeV}$, CMS-PAS-EXO-15-002, CERN, Geneva Switzerland (2015).

[62] ATLAS collaboration, Search for a new resonance decaying to a $W$ or $Z$ boson and a Higgs boson in the $\ell \ell / \ell \nu / \nu \nu+b \bar{b}$ final states with the ATLAS detector, Eur. Phys. J. C 75 (2015) 263 [arXiv: 1503.08089] [INSPIRE].

[63] CMS collaboration, Search for a massive resonance decaying into a Higgs boson and a $W$ or $Z$ boson in hadronic final states in proton-proton collisions at $\sqrt{s}=8 \mathrm{TeV}$, arXiv: 1506.01443 [INSPIRE].

[64] CMS collaboration, Search for massive $W H$ resonances decaying to $\ell \nu b \bar{b}$ final state in the boosted regime at $\sqrt{s}=8 \mathrm{TeV}$, CMS-PAS-EXO-14-010, CERN, Geneva Switzerland (2014). 
[65] ATLAS collaboration, Search for new resonances decaying to a $W$ or $Z$ boson and a Higgs boson in the $\ell \ell b \bar{b}, \ell \nu b \bar{b}$ and $\nu \nu b \bar{b}$ channels in pp collisions at $\sqrt{s}=13 \mathrm{TeV}$ with the ATLAS detector, ATLAS-CONF-2015-074, CERN, Geneva Switzerland (2015).

[66] CMS collaboration, Search for resonances decaying to dijet final states at $\sqrt{s}=8 \mathrm{TeV}$ with scouting data, CMS-PAS-EXO-14-005, CERN, Geneva Switzerland (2014).

[67] ATLAS collaboration, Search for new phenomena in the dijet mass distribution using pp collision data at $\sqrt{s}=8 \mathrm{TeV}$ with the ATLAS detector, Phys. Rev. D 91 (2015) 052007 [arXiv: 1407.1376] [INSPIRE].

[68] CMS collaboration, Search for resonances and quantum black holes using dijet mass spectra in proton-proton collisions at $\sqrt{s}=8 \mathrm{TeV}$, Phys. Rev. D 91 (2015) 052009 [arXiv: 1501.04198] [INSPIRE].

[69] ATLAS collaboration, Search for new phenomena in dijet mass and angular distributions from pp collisions at $\sqrt{s}=13 \mathrm{TeV}$ with the ATLAS detector, Phys. Lett. B 754 (2016) 302 [arXiv: 1512.01530] [INSPIRE].

[70] CMS collaboration, Search for narrow resonances decaying to dijets in proton-proton collisions at $\sqrt{s}=13 \mathrm{TeV}$, Phys. Rev. Lett. 116 (2016) 071801 [arXiv:1512.01224] [INSPIRE].

[71] A.D. Martin, W.J. Stirling, R.S. Thorne and G. Watt, Parton distributions for the LHC, Eur. Phys. J. C 63 (2009) 189 [arXiv:0901.0002] [inSPIRE].

[72] CMS collaboration, Search for heavy neutrinos and $W$ bosons with right-handed couplings in proton-proton collisions at $\sqrt{s}=8 \mathrm{TeV}$, Eur. Phys. J. C 74 (2014) 3149 [arXiv:1407.3683] [INSPIRE]. 